\title{
Constraints on Warm Dark Matter Models from the High Redshift Universe
}

\section{Zoltán Haiman* $\dagger$}

Princeton University Observatory, Ivy Lane, Princeton, NJ 08544, USA

E-mail: 'zoltandastro.princeton.edu'

\section{Rennan Barkana}

Canadian Institute for Theoretical Astrophysics, 60 St. George Street, Toronto, Ontario, M5S 3H8, CANADA

E-mail: 'barkana@cita.utoronto.ca'

\section{Jeremiah P. Ostriker}

Princeton University Observatory, Ivy Lane, Princeton, NJ 08544, USA

E-mail: 'jpo@astro.princeton edu'

ABstRACT: Warm Dark Matter (WDM) models have recently been resurrected to resolve apparent conflicts of Cold Dark Matter (DM) models with observations. Endowing the DM particles with non-negligible velocities causes free-streaming, which suppresses the primordial power spectrum on small scales. The choice of a root-mean-square velocity dispersion $v_{\mathrm{rms}, 0} \sim 0.05 \mathrm{~km} / \mathrm{s}$ at redshift $z=0$ (corresponding to a particle mass $m_{X} \sim 1$ $\mathrm{keV}$ if the WDM particles are fermions decoupling while relativistic) helps alleviate most, but probably not all, of the small-scale problems faced by CDM. An important side-effect of the particle velocities is the severe decrease in the number of collapsed halos at high redshift. This is caused both by the loss of small-scale power, and by the delay in the collapse of the smallest individual halos (with masses near the effective Jeans mass of the $\mathrm{DM})$. The presence of early halos is required in order (1) to host either early quasars or galaxies that can reionize the universe by redshift $z=5.8$, and (2) to allow the growth of the supermassive black hole believed to power the recently discovered quasar SDSS 10441215 at this redshift. We quantify these constraints using a modified Press-Schechter formalism, and find $v_{\mathrm{rms}, 0} \lesssim 0.04 \mathrm{~km} / \mathrm{s}$ (or $m_{X} \gtrsim 1 \mathrm{keV}$ ). If future observations uncover massive black holes at $z \gtrsim 10$, or reveal that reionization occurred at $z \gtrsim 10$, this could conclusively rule out WDM models as the solution to the small-scale crisis of the CDM paradigm.

\footnotetext{
${ }^{*}$ Speaker.

${ }^{\dagger}$ Hubble Fellow.
} 


\section{Introduction}

The currently favored model of hierarchical galaxy formation in a universe dominated by cold dark matter (CDM) has been very successful in matching observations of the density distribution on large scales. However, recently some small-scale shortcomings of this model have appeared, as summarized elsewhere in these proceedings (see also [i] for a recent review). Although the observational significance of the discrepancies is still disputed, and astrophysical solutions involving feedback may still be possible, the accumulating tension with observations has focused attention on solutions involving the particle properties of dark matter. Proposals have included self-interacting dark matter [iㅁ] adding a repulsive interaction to gravity [3ian

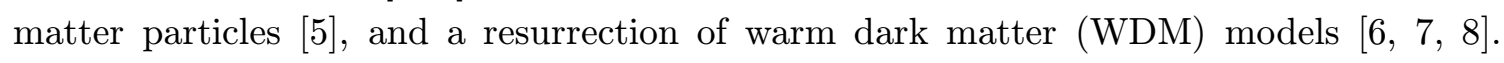
By design, a common feature of models that attempt to solve the apparent small-scale problems of CDM is the reduction of fluctuation power on small scales. In the CDM paradigm, structure formation proceeds bottom-up: the smallest objects collapse first, and they subsequently merge together to form larger objects. It then follows that the loss of small-scale power modifies structure formation most severely at the highest redshifts; in particular, the number of self-gravitating objects at high redshift is reduced.

A strong reduction in the abundance of high-redshift objects could be in conflict with observations. First, the lack of a Gunn-Peterson trough in the spectrum of the bright quasar SDSS 1044-1215 at redshift $z=5.8[\overline{9}]$ implies that the hydrogen in the intergalactic medium (IGM) was highly ionized prior to this redshift. The most natural explanation for reionization is photo-ionizing radiation produced by an early generation of stars or quasars [i] $1 \overline{0} \overline{0}]$. The sources of reionization reside in halos that have masses in the range corresponding to dwarf galaxies - the mass scale on which power needs to be reduced relative to CDM models. Second, if SDSS 1044-1215 is unlensed and radiating at or below the Eddington limit, its unusual intrinsic brightness implies that it is powered by an exceptionally massive black hole (BH). The growth of this $\mathrm{BH}$, out of a stellar remnant seed, requires [I] host halo be present at a sufficiently high redshift $(z \gg 5.8)$.

In this contribution, we briefly review the status of WDM models in resolving the problems of CDM, and then examine new constraints that arise on WDM models from the high redshift universe. We focus on WDM models, although similar constraints would apply to other modifications of the CDM paradigm that reduce the small-scale power. The cosmological parameters we adopt, based on present large scale structure data [i] $\overline{2}]$, are $\left(\Omega_{0}, \Omega_{\Lambda}, \Omega_{\mathrm{b}}, h, \sigma_{8}, n\right)=(0.3,0.7,0.045,0.7,0.9,1)$. The details of the study described here can be found in [i 13 in].

\section{Warm Dark Matter Models}

The WDM is assumed to be composed of particles of about $\sim 1 \mathrm{keV}$ mass (compared to $\sim 1 \mathrm{GeV}$ in $\mathrm{CDM}$, or $\sim 10 \mathrm{eV}$ in Hot Dark Matter models). The thermal velocities of the particles cause free streaming out of overdense regions, smoothing out small-scale fluctuations, leading to a small-scale cutoff in the linear power spectrum. In addition, the thermal 
velocities act similarly to pressure, and inhibit the growth of low-mass perturbations. One example of WDM is fermionic particles that decouple in the early universe while relativistic and in thermal equilibrium [1 in] . To produce a given contribution $\Omega_{X}$ to the cosmological critical density, the required particle mass $m_{X}$ is then determined by $m_{X} n_{X} \propto \Omega_{X} h^{2}$, where the present number density $n_{X}$ of WDM particles follows from their chosen r.m.s. velocities. This yields a relation between particle mass and r.m.s. velocity dispersion [is],

$$
v_{\mathrm{rms}}(z)=0.0437(1+z)\left(\frac{\Omega_{X} h^{2}}{0.15}\right)^{1 / 3}\left(\frac{g_{X}}{1.5}\right)^{-1 / 3}\left(\frac{m_{X}}{1 \mathrm{keV}}\right)^{-4 / 3} \mathrm{~km} \mathrm{~s}^{-1}
$$

where $g_{X}$ is the effective number of degrees of freedom of WDM. The comoving cutoff scale $R_{c}$, where free-streaming reduces the power spectrum to half of its value in CDM, is given by

$$
\begin{aligned}
R_{c} & =0.201\left(\frac{\Omega_{X} h^{2}}{0.15}\right)^{0.15}\left(\frac{g_{X}}{1.5}\right)^{-0.29}\left(\frac{m_{X}}{1 \mathrm{keV}}\right)^{-1.15} \mathrm{Mpc} \\
& =0.226\left(\frac{\Omega_{X} h^{2}}{0.15}\right)^{-0.14}\left(\frac{v_{\mathrm{rms}, 0}}{0.05 \mathrm{~km} / \mathrm{s}}\right)^{0.86} \mathrm{Mpc} .
\end{aligned}
$$

The length scale $R_{c}$ corresponds to a characteristic mass scale $M_{c}$,

$$
M_{c}=1.74 \times 10^{8}\left(\frac{\Omega_{0} h^{2}}{0.15}\right)\left(\frac{R_{c}}{0.1 \mathrm{Mpc}}\right)^{3} M_{\odot} .
$$

The predictions of WDM models can be expected to differ from CDM on scales below $R_{c}$ or $M_{c}$. In addition, it is useful to define an "effective Jeans mass" for WDM, at which the pressure corresponding to the r.m.s. particle velocities balances gravity,

$$
\begin{aligned}
M_{J} & =3.06 \times 10^{8}\left(\frac{g_{X}}{1.5}\right)^{-1}\left(\frac{\Omega_{X} h^{2}}{0.15}\right)^{1 / 2}\left(\frac{m_{X}}{1 \mathrm{keV}}\right)^{-4}\left(\frac{1+z_{i}}{3000}\right)^{3 / 2} M_{\odot} \\
& =4.58 \times 10^{8}\left(\frac{\Omega_{X} h^{2}}{0.15}\right)^{-1 / 2}\left(\frac{v_{\mathrm{rms}, 0}}{0.05 \mathrm{~km} / \mathrm{s}}\right)^{3}\left(\frac{1+z_{i}}{3000}\right)^{3 / 2} M_{\odot} .
\end{aligned}
$$

We have verified these scalings using spherically symmetric, one-dimensional collapse simulations [1] "pressure" of WDM. Although this effect is irrelevant in most discussions of WDM models at lower redshifts (due to the smallness of $M_{J}$ ), we find that it is important in the context of reionization. The inhibited growth of perturbations in the linear regime results in a delay in the final virialization epoch of the individual, low-mass halos, that first condense out in the universe.

\section{Summary of Current Observations}

The current status of WDM models in resolving various problems of CDM is summarized in Table ii:- Based on analytical arguments, as well as numerical simulations, WDM particles with a mass in the range $0.6 \mathrm{keV} \lesssim m_{X} \lesssim 1.5 \mathrm{keV}$ can strongly suppress the number of 


\begin{tabular}{|c|c|c|c|}
\hline WDM mass & Status & References & \\
\hline Milky Way satellites & $0.6-1.5 \mathrm{keV}$ & $\sqrt{ }$ & 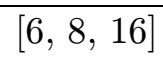 \\
\hline Halo concentration & $0.6-1.5 \mathrm{keV}$ & $\sqrt{ }$ & 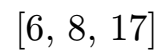 \\
\hline Inner density profile & $<0.6 \mathrm{keV} ?$ & $?$ & {$[\overline{\bar{\theta}}, \overline{1}, \overline{1}, \overline{1}, \overline{1}]$} \\
\hline No dwarfs in voids & $<1.5 \mathrm{keV}$ & $?$ & \\
\hline Angular momentum & $0.5-0.8 \mathrm{keV}$ & $\mathrm{x} ?$ & {$[\underline{\overline{1}}, 1,1]$} \\
\hline
\end{tabular}

Table 1: Scorecard of WDM models. Note that WDM particles with $m_{X} \lesssim 750 \mathrm{eV}$ would contradict the power spectrum of the $\operatorname{Ly} \alpha$ forest $\left[1 \overline{1} \overline{5}_{n}\right.$. A similar limit follows from the maximum observed phase space density in cores of dwarf spheroidals [i $[\bar{i} i]$.

satellite halos of the Milky Way, and produce low halo concentration parameters for both

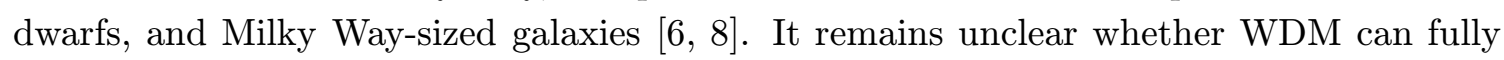
resolve some other, related problems. One of the major successes of the CDM paradigm is the interpretation of the statistics of the Ly $\alpha$ forest. An analysis of the Ly $\alpha$ forest power spectrum at redshift $z \approx 3$ finds that WDM models with $m_{X}<0.75 \mathrm{keV}$ would spoil this success [1] $[1 \overline{1} \overline{1}$. A similar limit from the maximum observed phase space density in dwarf spheroidal galaxies. To solve some of the problems with CDM, lower masses might be needed. In existing simulations for $m_{x}>0.6 \mathrm{keV}$, the inner slope of the density profiles of normal disk galaxies do not appear to flatten sufficiently to produce the observed, nearly constant cores, although these simulations do not yet probe the relevant innermost regions reliably [iㅁ] $]$. A similar situation arises with two other CDM problems. The solution of the long-standing angular-momentum problem in galaxy formation might be solved if "subclumps" in the protogalaxy's halo were eliminated (CDM models produce too small disks, thought to be attributable to angular momentum transfer from the infalling gas to the DM subclumps). Although WDM does suppress halo substructure, the required WDM mass is estimated to be $0.5-0.8 \mathrm{keV}\left[\bar{q}_{-1}\right]$. Furthermore, a simulation that artificially turns off angular momentum transfer between gas and DM halo subclumps reveals that

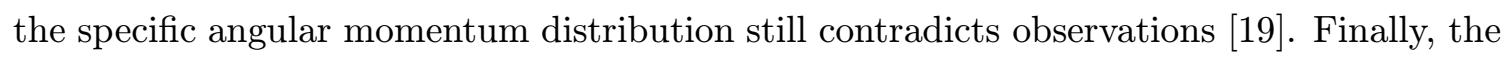
absence of dwarf galaxies in voids, a difficulty of CDM [i] $1 \overline{8}]$, is found to be eased in a

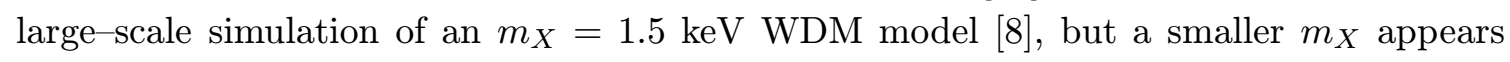
necessary to match the observations in detail.

\section{Constraints from Reionization}

As emphasized in section $\S 1$, WDM models reduce the abundance of high-redshift halos, which could lead to conflicts with observations. Note that in general, the differences between WDM and CDM models are amplified at high redshifts. In a study based on a modified version of the extended Press-Schechter theory, we have quantified constraint on the velocities and masses of WDM particles from reionization, and from the presence of a supermassive BH in the bright $z=5.8$ quasar SDSS 1044-1215. The main features of our models are as follows: 
1. The effect of free streaming on the power spectrum was included using a fit to the numerical transfer function, obtained from a Boltzmann code.

2. The effect of WDM velocities on the growth of perturbations was included using spherically symmetric, one-dimensional hydrodynamical simulations.

3. The WDM halo mass function was computed using Monte Carlo-generated halo "merger trees". A moving barrier $\left(\delta_{c} \geq 1.69\right)$ was adopted to generalize (an improved) Press-Schechter theory to the case of mass-dependent collapse times. Our semianalytic halo mass function was demonstrated to agree with numerical simulations at low redshift over the halo masses of interest [isin.

4. We assume that a fraction $f_{*}$ of baryons turns into stars in halos with virial temperatures $T_{\text {vir }} \geq 10^{4} \mathrm{~K}$ (necessary for efficient cooling $[\overline{2} \overline{2} \overline{0} \mid]$ ). We assume a Scalo (1998) stellar IMF (producing $\approx 4000$ ionizing photons per baryon), and that a fraction $f_{\text {esc }}$ of the ionizing photons escape into the IGM. We parameterize our models by the product $\epsilon_{*} \equiv f_{*} f_{\text {esc }}(=0.01$ in our standard model $)$, consistent with the ionizing background inferred from the proximity effect at redshift $z \approx 3$. [i2 $\overline{1} \overline{1}]$

5. The mean clumping of the ionized gas in the IGM is parameterized by a single constant $C \equiv\left\langle n_{\mathrm{H}}^{2}\right\rangle / \bar{n}_{\mathrm{H}}^{2}(=10$ in our standard model $)$.

\section{Conclusions}

If high-redshift galaxies produce ionizing photons with an efficiency similar to their $z=3$ counterparts $\left(\epsilon_{*} \sim 0.01\right)$, reionization by redshift $z=5.8$ places a limit of $m_{X} \gtrsim 1.25 \mathrm{keV}$ $\left(v_{\mathrm{rms}, 0} \lesssim 0.03 \mathrm{~km} / \mathrm{s}\right)$ on the mass of the WDM particles. This limit is somewhat stronger than the limit inferred from the statistics of the Ly $\alpha$ forest (which yields $m_{X} \gtrsim 0.75 \mathrm{keV}$; [1] uncertainty in current measurements of the stellar contribution to the ionizing intensity at $z=3[\overline{2} \overline{1} \overline{1}]$ (which we can use to normalize our models). We also find that the existence of $\mathrm{a} \approx 4 \times 10^{9} \mathrm{M}_{\odot}$ supermassive black hole at $z=5.8$, powering the quasar SDSS 10441215 (assuming it is unlensed and radiating at or below the Eddington limit), yields the somewhat weaker, but independent constraint $m_{X} \gtrsim 0.5 \mathrm{keV}$ (or $v_{\mathrm{rms}, 0} \lesssim 0.10 \mathrm{~km} / \mathrm{s}$ ), if this $\mathrm{BH}$ acquired most of its mass accreting at the Eddington rate. Finally, we also find that WDM models with $m_{X} \lesssim 1 \mathrm{keV}\left(v_{\mathrm{rms}, 0} \gtrsim 0.04 \mathrm{~km} / \mathrm{s}\right)$ produce a low-luminosity cutoff in the high- $z$ galaxy luminosity function which is detectable with the Next Generation Space Telescope. Such an observation would directly break the degeneracy in the reionization redshift between low ionizing-photon production efficiency and small WDM particle mass. The constraints derived here will tighten considerably as observations probe still higher redshifts, offering increasingly stringent tests of models with diminished small-scale power, exemplified by WDM.

ZH acknowledges support from a Hubble Fellowship, and RB acknowledges support from CITA and from Institute Funds (IAS, Princeton). 


\section{References}

[1] Sellwood, J., and Kosowsky, A., in Gas and Galaxy Evolution, eds. Hibbard, Rupen and van Gorkom, in press, astro-ph/0009074 (2001).

[2] Spergel, D. N., and Steinhardt, P. J., Phys. Rev. Lett. 84, 3760 (2000).

[3] Goodman, J., New Astronomy 5, 103 (2000).

[4] Peebles, P. J. E., ApJ 534, 127 (2000).

[5] Hu, W., Barkana, R., and Gruzinov, A., Phys. Rev. Lett. 85, 1158 (2000).

[6] Colín, P., Avila-Reese, V., and Valenzuela, O., ApJ 542, 622 (2001).

[7] Sommer-Larsen, J., and Dolgov, A., Ap. J. submitted, astro-ph/9912166 (1999).

[8] Bode, P., Ostriker, J. P., and Turok, N., Ap. J., 556, 93 (2001).

[9] Fan, X., et al., Astron. J. 1210, 1167 (2000).

[10] Barkana, R., and Loeb, A., Physics Reports, 349, No.2 (2001).

[11] Haiman, Z., and Loeb, A., ApJ 552, 459 (2001).

[12] Bahcall, N., et al., Science 284, 1481 (1999).

[13] Barkana, R., Haiman, Z., and Ostriker, J. P., Ap. J., 558, 482 (2001).

[14] Kolb, E. W., and Turner, M. S., The Early Universe, Addison-Wesley (1990).

[15] Narayanan, V. K., Spergel, D. N., Davé, R., and Ma, C., Ap. J. 543, L103 (2000).

[16] White, M., and Croft, R., Ap. J. 539, 497 (2000).

[17] Hogan, C. J., and Dalcanton, J. J., Phys. Rev. D. 62, 3511 (2000).

[18] Peebles, P. J. E. Ap. J. 557, 495 (2001).

[19] Bullock, J. S. et al. 2001, ApJ submitted, preprint astro-ph/0011001.

[20] Haiman, Z., Abel, T., and Rees, M. J., ApJ 534, 11 (2000).

[21] Bajtlik, S., Duncan, R. C., and Ostriker, J. P., ApJ 327, 570 (1988). 